\title{
EN EL MUNDO CON OTROS: UNA EXPERIENCIA DE HOSPITAL DE DÍA DE BUENOS AIRES/ARGENTINA
}

\author{
NO MUNDO COM OUTROS: UMA EXPERIÊNCIA DO DIA HOSPITALAR DE BUENOS \\ AIRES / ARGENTINA
}

\author{
SHARING THE WORLD WITH OTHERS: BUENOS AIRES, ARGENTINA A DAY \\ HOSPITAL EXPERIENCE
}

\author{
Gisela Súcari ${ }^{1}$ \\ Universidad de Buenos Aires - UBA \\ María Inés Borrás² \\ Universidad de Buenos Aires - UBA \\ Pablo Damiano ${ }^{3}$ \\ Universidad de Buenos Aires - UBA \\ Adriana Giordano 4 \\ Universidad de Buenos Aires - UBA \\ Magalí Nogueras 5 \\ Universidad de Buenos Aires - UBA \\ Paula Raimondi 6 \\ Universidad de Buenos Aires - UBA \\ Soledad Sturla7 \\ Universidad de Buenos Aires - UBA
}

\footnotetext{
${ }^{1}$ Hospital de Emergencias Psiquiátricas Torcuato de Alvear. Argentina. Lic. en Trabajo Social pela Universidad de Buenos Aires. E-mail: gsucari@hotmail.com

2 Hospital de Emergencias Psiquiátricas Torcuato de Alvear. Argentina. Lic. En Psicología pela Universidad de Buenos Aires (UBA). E-mail: mariainesborras@gmail.com

${ }^{3}$ Hospital de Emergencias Psiquiátricas Torcuato de Alvear. Argentina. Médico pela Universidad de Buenos Aires (UBA). Especialista en psiquiatría. E-mail: pablofdamiano@gmail.com

${ }^{4}$ Hospital de Emergencias Psiquiátricas Torcuato de Alvear. Argentina. Licenciada en Psicología pela Universidad de Buenos Aires (UBA). E-mail: adrigior57@yahoo.com.ar

${ }^{5}$ Hospital de Emergencias Psiquiátricas Torcuato de Alvear. Argentina. Licenciatura en Terapia Ocupacional pela Universidad de Buenos Aires (UBA). E-mail: magalinogueras@hotmail.com

${ }^{6}$ Hospital de Emergencias Psiquiátricas Torcuato de Alvear. Argentina. Médica pela Universidad de Buenos Aires (UBA). Especialista en psiquiatría Infanto Juvenil. E-mail: paularaimondi@yahoo.com

7 Hospital de Emergencias Torcuato de Alvear. Argentina. Médica pela Universidad de Buenos Aires (UBA). Especialista en psiquiatría. E-mail: soledadcsturla@gmail.com
} 


\section{Resumen}

Este escrito es el intento de de compartir una experiencia de trabajo en un Hospital de Día situado en la ciudad de Buenos Aires - Argentina. Surge del trabajo realizado a partir de repensar conceptos clásicos relacionados al dispositivo, como por ejemplo: resocialización, rehabilitación, "espacio de ficción" entre otros; a partir de los interrogantes surgidos desde la clínica. Esto nos llevó a abrir espacios de talleres a la comunidad y de este modo propiciar el encuentro con otros.

Palabras Clave: Lazo social; Ser en el mundo; Hospital de Día abierto

\section{Resumo}

Este escrito é a tentativa de compartilhar uma experiência de trabalho em um Hospital de Dia localizado na cidade de Buenos Aires - Argentina. Surge do trabalho de repensar conceitos clássicos relacionados ao dispositivo, como: ressocialização, reabilitação, "espaço ficcional" entre outros. E nos interrogarmos sobre nossas tarefas diárias com base nas questões levantadas na clínica. Isso nos levou a abrir espaços para oficinas para a comunidade e, assim, incentivar o encontro com os outros.

PalavrasChave: Laço social; Ser no mundo; Hospital do Dia Aberto.

\section{Abstract}

This is an attempt to share a work experience in a Day Hospital located in the city of Buenos Aires Argentina. It arises from the work of rethinking classic concepts related to the topic, such as: resocialization, rehabilitation, "fictional space" among others. And we inquired about our daily tasks based on the issues raised at the clinic. This has led us to open spaces for workshops for the community and thus encourage the encounter with others.

Keywords: Social link; Dasein; A Day Hospital open to community.

\section{INTRODUCCIÓN}

El presente es un relato de experiencia. Quienes formamos parte del equipo de Hospital de Día turno tarde del Hospital de Emergencias Psiquiátricas Torcuato de Alvear. (HEPTA), tomamos en determinado momento, la decisión de volver a pensar el dispositivo. Esto nos llevó a revisar y trabajar en relación a dos conceptos; resocialización y rehabilitación, ambos postulados como fundamentos del mismo.

El Hospital de Emergencias Psiquiátricas "Torcuato de Alvear" se encuentra ubicado en la capital de la República Argentina, la Ciudad Autónoma de Buenos Aires (Av. Warnes 2630), en una zona geográfica conocida como "la isla": ubicado detrás del cementerio de Chacarita, enfrente del terreno que anteriormente ocupaba el albergue Warnes donde actualmente funciona el supermercado Carrefour. El hospital es lindero al Hogar San Martín de adultos 
mayores y tiene como "telón de fondo" las vías del ferrocarril San Martín y Urquiza que delimitan los alcances de "la isla".

El hospital comenzó su construcción en 1908 abriendo sus puertas en 1909. Cabe destacarse que fue creado en sus inicios como Hospital General de Agudos siendo esta su especialidad hasta 1977, año en el cual es cerrado y desmantelado (como otros hospitales públicos) por la dictadura cívico- militar instalada en Argentina en el período 1976-1983. En ese año, y restaurando la democracia, el Hospital Torcuato de Alvear reabre sus puertas a la comunidad, transformado en un efector especializado en salud mental pensado exclusivamente para padecimientos agudos. Entre el periodo 1983-1990 funcionó como "Unidad de Emergencias Psiquiátricas" dependiente del hospital Pirovano. Se fundó así el primer efector de Salud Mental de la Argentina destinado a emergencias, siendo su característica principal el carácter interdisciplinario del abordaje de la locura en su estado agudo, clásicamente hasta entonces encarado por la Psiquiatría. A partir de 1990 se establece su autonomía del H. Pirovano, pasando a ser nombrado como Hospital de Emergencias Psiquiátricas Torcuato de Alvear (HEPTA).

El Hospital forma parte del subsistema de salud público de la Ciudad Autónoma de Buenos Aires, dependiente del Ministerio de Salud del Gobierno de la Ciudad. De acuerdo a la Ley Básica de Salud N¹53, el Sistema de Salud "está integrado por el conjunto de recursos de dependencia estatal, de la seguridad social y privada que se desempeñan en el territorio de la Ciudad Autónoma de Buenos Aires". Se caracteriza por ser un efector monovalente, es decir abocado exclusivamente al abordaje de la salud mental. Como tal, es integrante del Sistema de Salud Mental de la Ciudad, que de acuerdo a la Ley de Salud Mental de la Ciudad de Buenos Aires $N^{\circ} 448$, está "constituido por los recursos del Sistema de Salud Mental de los subsectores estatal, de seguridad social y privado que se desempeñan en el territorio de la Ciudad", y al ser un efector de orden estatal funciona integrando la Red de Atención del Sistema de Salud Mental.

Retomando la intención de describir esta nueva experiencia del Servicio de Hospital de Día Turno Tarde, no es sencillo fechar el comienzo de esta labor. Resulta ineludible reconocer el trabajo realizado por todos aquellos que nos precedieron en la tarea y poder recibir la enorme influencia de una herencia que es la suya. Usamos la palabra herencia teniendo en consideración el valor que a esta palabra le otorga Derrida y Roudinesco (2009), para quién la figura del heredero es la de aquél que para poder recibir una herencia debe tomar la decisión 
de elegir, de salvar y destruir lo que le llega. Para ser fiel a una herencia, la de un pensamiento, tiene la responsabilidad de ser infiel en tanto debe criticarlo, discutirlo. Discusión entablada desde una posición fiel-infiel al mismo tiempo. Discusión imposible en algún punto. Discusión que toma relevo del movimiento de ese pensamiento en el mismo momento que lo destruye. Tendremos esto en cuenta constantemente en cada uno de los desarrollos que siguen.

Entonces, al intentar compartir una experiencia que es la nuestra, es necesario, fiel e infielmente, ubicar algún inicio, arbitrario, de la misma. De la experiencia de la cual se quiere dar cuenta en estos textos. Con todas estas precauciones y asumiendo la, por un lado, gigantesca y por otro lado forzosa, limitación antes declarada, vamos a ubicarnos en los tiempos que corrían alrededor del año 2014.

Quienes trabajábamos en el dispositivo podíamos dar cuenta de la potencia del mismo. Esto era un hecho innegable, aunque en muchos casos tomábamos conciencia también de que no sabíamos, en aquel momento, dónde es que esa potencia se sostenía realmente. Comenzaban a no alcanzarnos las explicaciones que veníamos repitiéndonos. Para empezar, en muchos y variados aspectos, el Hospital de Día donde trabajábamos, no se parecía en nada a las descripciones clásicas.

Después de realizado cierto trabajo (trabajo realizado con aquellos a quienes en primer lugar queremos dar nuestro agradecimiento por todo lo que nos enseñaron, aquellos a quienes atendimos, los pacientes) creemos hoy estar en condiciones de poder realizar algunas afirmaciones al respecto. Es esto, quizás, lo más relevante en este relato y es un punto que volveremos a tocar más adelante.

\section{CONDICIONES}

Existen algunos requerimientos que tienen que ver con la capacidad del dispositivo de sostener al paciente. Estos se relacionan con ciertas características del mismo. Las más importantes son:

- Se trata de un tratamiento ambulatorio. Es importante entonces que el paciente cuente con un lugar en el cual residir. También, que se cuente con algún grado de contención socio-familiar.

- La carga horaria (lunes a viernes media jornada). Esto lleva a considerar la accesibilidad del tratamiento en lo que se refiere a distancias (tiempo y costo económico)

- La aceptación de normas en relación a horarios, actividades y de convivencia. 
Estas condiciones son importantes y están vinculadas a un concepto de accesibilidad al sistema de salud. Un concepto de accesibilidad más complejo que el simplemente relacionado a la llegada del paciente al sistema de salud. Un concepto de accesibilidad que habla de la capacidad del sistema de sostener al paciente en el tiempo. Si bien son requerimientos importantes, lo son con el objeto de dar lugar a los tratamientos. Por lo cual no los entendemos como criterios de exclusión y en la medida de lo posible son requerimientos flexibles y que intentamos pensar en cada caso singular. Algo que nos orienta en este pensar es intentar considerar en cada caso "qué es lo que podemos ofrecer".

\section{INDICACIONES}

Por otro lado, está aquello sobre lo cual este dispositivo permite trabajar en forma preferencial y específica. Se trata de aquello acerca de lo cual decíamos en la introducción que podíamos hoy afirmar algo. Pero habrá que llegar a eso de a poco. Comencemos.

Trabajar en la resocialización y la rehabilitación. Las indicaciones clásicas. Podemos decir que de ellas, la inmediatamente generosa, la que inmediatamente se ofrece a la crítica, es la rehabilitación.

Es con este concepto de rehabilitación que se relaciona una idea que circula en el imaginario social. Un aspecto importante del dispositivo tiene que ver con la realización de actividades. En hospital de día "se hacen talleres". Hay horarios, actividades, y hasta en algún caso, la idea de que se pudiera no solo ocupar el tiempo vacío de aquél que es derivado, no solo "hacer algo" sino también que quizás se pueda "hacer algo útil", incluso hasta aprender algún oficio. Esta idea casi siempre tiene alguna incidencia en las derivaciones.

Cabe mencionar además en este momento que dichas derivaciones son por regla general pensadas, indicadas, no por quien llega de esta manera "derivado", sino por algún otro (profesional, familiar, etc.). Los pacientes llegan al hospital de día casi siempre derivados por algún otro. Por este hecho, de manera estructural e inevitable, siempre en la derivación va a aparecer en menor o mayor medida algo de lo que se ha identificado como la "demanda social". Muchas veces esta demanda está orientada por una idea también normativa de lo que es la rehabilitación.

Es este un punto en particular en el que es necesario hacer referencia a quienes se detuvieron antes a pensar el problema que el concepto de rehabilitación plantea. Esta es la 
herencia que antes mencionábamos. Hacer referencia en este punto a nuestra herencia supone pronunciar el nombre de Rodolfo luorno.

Desde los comienzos del trabajo en el hospital de día del HEPTA, épocas en que luorno fuera coordinador, se realizó una labor en relación a ese punto que podemos situar entre la demanda social y la rehabilitación. Es de los frutos de aquel trabajo que hemos recibido algo muy concreto en herencia. En el proceso de admisión de un paciente, hoy, de un modo naturalizado, trabajamos para intentar modificar algo de esta demanda social. Para intentar dar la palabra a quien en principio es traído, derivado, enviado, por otros. Es de la época y el trabajo de Rodolfo luorno que hemos recibido esa herencia, la de pensar que la admisión sea "la admisión de un decir". De un decir particular de quien llega. De un decir que en la enorme mayoría de los casos no está presente de entrada.

Es por aquello de lo que somos herederos que entonces la admisión deja de ser solamente un momento burocrático, en el que se recaban los datos acerca de la "indicación" y "criterios de admisión" exigidos por el dispositivo y pasa a ser la apuesta a que alguien pueda, quizás, iniciar tratamiento tomando la palabra.

Muchas veces esto plantea todo un trabajo de construcción junto con el paciente de un decir propio. Todo un trabajo entonces que se realiza en la admisión para, con mayor o menor éxito, dar lugar a la palabra de quien llega inicialmente más bien en una posición de "ser hablado".

\section{FRAGMENTOS}

- "Es necesario (...) distinguir la demanda social que hace operar al Hospital de Día, de la demanda de tratamiento que quien consulta realiza”.

- “ ¿(...) ...se puede operar más allá de satisfacer una demanda social?"

El problema a mi entender, consiste en definir qué entendemos por rehabilitación y resocialización (...) ¿escuchamos que el sujeto es lo que responde a determinaciones sociales imperantes y que la rehabilitación y resocialización en consecuencia se dirigen y se determinan desde un discurso amo que convalida qué es lo normal y eficiente en cada periodo de la historia?

Tanto la rehabilitación como la resocialización hay que pensarlas en relación a la demanda singular que cada sujeto pueda efectuar durante el proceso de su tratamiento (IUORNO, 2004, p. 79). 


\section{SER EN EL MUNDO: SER CON OTROS}

..." En razón de este concomitante "ser en el mundo" es el mundo en cada caso ya siempre aquel que comparto con otros"

(HEIDEGGER, 2009)

So pretexto de poder contar nuestra experiencia y en contra de la inercia que plantea la estructura institucional, hicimos lugar a un espacio de trabajo donde pensar la clínica y los tratamientos. Pensarlos desde: "lo público", "el lazo social", el "nosotros". A partir de otra concepción de lo público encontramos alguna apoyatura en las ideas de Heidegger en "El ser y el tiempo" para pensar los tratamientos menos desde los espacios individuales que desde los espacios comunitarios. Este texto, entre otros, nos ayudó a darle espacio a otros conceptos para pensar el dispositivo. En particular, las nociones de "ser en el mundo" y "ser con otros", nos ayudan a pensar la especificidad del hospital de día como un tratamiento del en y del con.

$\mathrm{Si}$ bien es algo que puede parecer obvio, para nosotros fue un redescubrimiento. Un asomarnos a la posibilidad de pensar que los órganos enfermos son ese con otros y ese en el mundo, esas dimensiones de la vida de alguien.

Se realiza en este dispositivo de hospital de día un trabajo intencional y explícito sobre el lazo social, el vínculo con otros. $\mathrm{Y}$ un tratamiento, un trabajo, mediante espacios de intercambio dentro y fuera del dispositivo de hospital de día y aún, dentro y fuera del hospital. En el dispositivo y en el afuera. Son los pacientes quienes nos orientan a dejar de pensar el dispositivo como una prótesis para intentar pensar además, el hospital de día como "un adentro que sea un afuera". Dejar de pensar entonces en oposición. Es ante todo un tratamiento, un trabajo, con otros. Se trata entonces de un tratamiento, de un trabajo, en el mundo.

La rehabilitación puede pensarse como un trabajo que responde a un para en el mundo, entendiendo así a la rehabilitación como una forma de respuesta a la demanda social de la "utilidad del sujeto". Según la concepción clásica rehabilitar se asocia a volver a habilitar "al loco" para que vuelva al mundo, (re)habilitarlo hasta y para que produzca. Tras repasar con actitud crítica esta concepción de la rehabilitación, en el hospital de día, comenzamos a pensar los tratamientos como un trabajo a realizar en el marco de un dispositivo que tiene como característica el hecho de trabajar desde y hacia con otros y desde y hacia en el mundo. 
Y entonces nos cabe la oportunidad, quizás, de pensar lo público atentos a otra resonancia que aquella que nos viene de la oposición público-privado. Y pensar lo público con otra estructura que la sostenida bajo aquella oposición permitiría, quizás, otra vez quizás, pensar lo público como otra cosa.

¿Cómo pensar lo público sin la referencia dada por el facilismo de la oposición a lo privado? Quizás, y ya van tres, lo público como dimensión de lo humano. ¿Fue así como comenzamos a pensar el nosotros?

Lo público, el hospital público, la salud pública, la escuela pública, cobran de repente otro sentido. El trabajo desde y hacia con otros. El trabajo desde y hacia en el mundo, lo humano como parte fundamental del nosotros, nosotros hacedores de lo público.

Estos enunciados son, sobre todo un punto de llegada y un punto de partida. Acá podemos ver que, al ser dos puntos, partida y llegada, hay un desde y un hacia, en constante movimiento y transformación. Estos enunciados son también un punto de llegada y un punto de partida. Son un hacia y un desde. Podríamos decir que se verifica en la afirmación misma de lo que quieren decir, o mejor, en la forma que toma esta afirmación, el nutrirse de una experiencia. La experiencia de un trayecto. Estas proposiciones son, entonces, provisionales en tanto corresponden a un trabajo en curso, a un trabajo abierto. Y la pretensión de que también lo abierto tenga lugar en la estructura. La pretensión de sostener una abertura, “...abertura del lugar que da lugar a lo que no tiene lugar" (NANCY, 2013).

\section{¿INTERDISCIPLINA? CONSIDERACIONES ACERCA DE LA OBEDIENCIA}

Existe un desarrollo clásico que ubica el término interdisciplina en un contexto. Multidisciplina - Interdisciplina - Transdisciplina, son términos que así ordenados hablan de un grado de complejidad que va creciendo en cuanto a la relación entre diversos campos del conocimiento que en la realidad se encuentran teniendo que convivir. Hay mucho material al respecto.

El camino que elegimos tomar va en la dirección contraria. Vamos a intentar dirigir nuestro pensar hacia donde nos orienta la palabra disciplina.

En el campo del conocimiento la idea de que en el mundo existe un cierto orden es un supuesto necesario. Es este supuesto, explicitado por el pensamiento cartesiano, el que hace posible entonces abordar el conocimiento del mundo (o de cualquier totalidad) dividiéndolo en partes y analizando y conociendo cada una de estas partes diferentes. Estas divisiones del 
mundo serán abordadas por diferentes ramas del conocimiento. Cada una de ellas recibe posteriormente el nombre de disciplina. Esto forma parte del paradigma de la modernidad, basado en la hegemonía de la razón. El tipo de conocimiento coherente con todo esto es el conocimiento científico.

En principio, el concepto de disciplina supone el de un orden, con leyes, reglas y normativas, con jerarquías y subordinaciones. El orden, entonces, en la base de la disciplina. Importa también señalar la intención detrás de este conocimiento: el dominio del mundo.

Pensar la interdisciplina obliga a pensar la disciplina. Orden. Subordinaciones y jerarquías. Dominio. Disciplinamiento y gobernabilidad. A no dejar de lado los aspectos políticos del término disciplina.

Imposible dejar de escuchar las resonancias que vinculan la producción de conocimiento y la penetración del capitalismo en el mundo. El dominio del mundo como ideal de la modernidad, las tecnologías de disciplinamiento y gobernabilidad. La obediencia.

\section{FRAGMENTOS $^{8}$}

- Primer tiempo. Fuera de disciplina.

- Extrañamente y para sorpresa de varios, comenzamos trabajando con la voz. No la voz como objeto de una teoría desde la cual se pueden seguramente decir muchas cosas. No me refiero a un trabajo teórico acerca de la voz.

- No.

- Cuando digo que trabajamos con la voz

- digo que el trabajo fue con la voz.

- Con la de cada uno.

- Con esa con la que cada uno cuenta

- (y no).

- El material, la materia con la que trabajamos fue la voz.

8 Fragmentos de Conversasiones mantenidas en el curso: "Fundamentos de Hospital de Día. Una política”. 2015. 
- Lo que en un tiempo previo fue "intentar pensar con las herramientas de otro lenguaje, la música" se transformó en otra cosa. En ese primer tiempo de trabajo, en el que fuimos acompañados por un músico, sucedió, además de aquello que más o menos habíamos planeado, otra cosa. Algo de lo no calculado fue teniendo lugar desde el inicio.

- Diversos trabajadores de la salud mental, de diferentes disciplinas, nos encontramos de repente fuera de las seguridades que a cada uno brinda la disciplina propia. Fuera incluso de cualquier posibilidad de ninguna (políticamente correcta) interdisciplina.

- Ninguna interdisciplina.

- Lo que apareció tuvo que ver más bien con ese "fuera". En primer lugar se trató de un espacio. Un espacio fuera.

- Cómo no ver hoy, luego de haber atravesado esta experiencia, que el trabajo estaba decididamente orientado allí donde nos encontrábamos más desorientados. Perdidos todos los puntos firmes, a riesgo de ridículos varios, apareció con ese espacio afuera, una posibilidad para el otro. Y con ella, una primer posibilidad para el diálogo."

Si la interdisciplina es la articulación de saberes provenientes de diferentes disciplinas, el riesgo es que en la práctica se termine dando más bien aquello que se llama multidisciplina o polidisciplina, en la cual se produce una suma de efectos provenientes de diferentes disciplinas pero sin que se produzca realmente algún "diálogo" entre las mismas. Un equipo de trabajo interdisciplinario no pocas veces, en no pocos momentos, se reduce tan solo a un grupo de profesionales de diferentes disciplinas con un acuerdo entre ellos acerca de qué cosa le corresponde y qué cosa no, a cada uno. Es necesario reconocer que en no pocos momentos esto nos ha sucedido y nos sucede. Inevitable. Quizás la cuestión pase por el hecho de que no sea así siempre. Que no sea solamente de este modo. Importa la intención, el intento, de que se produzca un diálogo. De que también se produzca un diálogo.

En este sentido, un descubrimiento. El diálogo, cuando apareció, no lo hizo desde ni entre disciplinas. Cuando apareció, lo hizo fuera de los límites de cualquier disciplina. ¿De cualquier obediencia?

\section{EL HOSPITAL DE DÍA ABIERTO}

Fue en parte debido a que las problemáticas sociales complejas con las que nos encontramos incluyen una fragilidad de los soportes relacionales que nos orientamos al trabajo 
en relación a aquello que denominamos el lazo, el lazo social. Fue por esto que se trabajó para propiciar espacios de encuentro que motiven a restituir algo de ese lazo social. De esta forma, la intervención en el espacio grupal permite trascender el plano de las necesidades materiales hacia problemas vinculados a lo subjetivo y relacional.

Fueron los pacientes quienes nos orientaron a dejar de pensar el hospital de día como un dispositivo protésico, en el sentido en que al menos en parte, por momentos, veníamos pensándolo. Esto también formaba parte de alguna herencia.

"Cifrado así, el tratamiento en un Hospital de Día se diferencia del tratamiento ofrecido por otras instituciones por ser la puerta de entrada a lo comunitario, intentando reproducir en su interior una microsociedad con sentido terapéutico." (IUORNO, 2004, p. 78)

Noción de una microsociedad, cerrada y cuidada, un ámbito protegido donde aquellos que ingresaban, con la ayuda profesional adecuada (y aquí es donde la ayuda profesional muchas veces tomaba la forma de lo protésico) pudieran ejercitarse en desarrollar determinados vínculos para más tarde, una vez rehabilitados en esta posibilidad, algo de esto pudiera ser aplicado afuera, en la verdadera sociedad.

Noción donde la rehabilitación (como respuesta a la demanda social) domina el campo y se entiende aún la resocialización bajo su óptica. Noción que culmina en los talleres de psicoeducación o de habilidades sociales (más allá de que en muchos casos este tipo de terapéuticas tengan eficacia en sus efectos, vale la pena evidenciar los supuestos en los que asientan).

Fueron los pacientes, decíamos, quienes nos orientaron en el camino de dejar de pensar el dispositivo como una microsociedad cerrada, como un espacio cerrado dentro del cual se podía brindar como tratamiento la posibilidad de sostener la ficción de una comunidad imposible por fuera. Fueron ellos quienes rompieron el cerco que el marco teórico que sosteníamos exigía.

Comenzamos a pensar espacios donde el lazo, el vínculo, dejara lugar al extranjero, al otro. Comenzaron a aparecer espacios nuevos en el Hospital de Día, como el "patio de visitas", donde por ejemplo fuera posible el tiempo compartido con pacientes en tratamiento en otros servicios. Aparecen los extraños, los extranjeros, los otros. Otros ajenos al hospital de día (administrativamente ajenos). 


\section{Y DE REPENTE NOS ENCONTRAMOS PENSANDO QUE PODÍA HABER EN EL HOSPITAL DE DÍA ESPACIOS DONDE FUERA POSIBLE EL VÍNCULO CON OTROS. (!!!!)}

Por este camino llegaron, luego del patio de visitas, los talleres abiertos. Tomamos la decisión de que algunos de los talleres que se realizaban en el dispositivo fueran abiertos. Abiertos no solo a pacientes de otros servicios, sino a quien quisiera participar. Profesionales y no profesionales de la comunidad hospitalaria participaron así del taller de plástica, de movimiento, de teatro. Comenzamos a pensar en talleres en hospital de día, abiertos a la comunidad $^{9}$.

Y luego de los talleres abiertos, se abre el espacio entre. El espacio que queda libre entre talleres, entrevistas, asamblea, etc. El espacio que se encuentra menos formalizado en el dispositivo, pero que vamos viendo tiene una potencia enorme en cuanto a los efectos. El espacio por el que transcurren muchos pacientes de manera preferencial en el dispositivo. Esos pacientes, algunos, que vienen, que no faltan, pero que no ingresan a los talleres. Se quedan afuera. Toman mate a veces en grupos otras veces solos. Vienen para quedarse afuera. Dejamos de intentar forzar un ingreso y salimos. Nuestra atención se orienta decididamente hacia lo abierto y el afuera. No ya la microsociedad cerrada, sino un hospital de día abierto. Empezamos a hablar y a pensar el hospital de día como un espacio de características particulares, "un adentro que sea un afuera"10.

Los talleres abiertos, por un lado, enriquecen el trabajo en el dispositivo en general, y por otro permiten ofrecer una opción para aquellos que no realizan su tratamiento formal en el hospital de día. Nos referimos a quienes no tienen su equipo tratante en este dispositivo.

El cronograma de talleres abiertos es dinámico. Actualmente em 2018 contamos con los siguientes:

\section{Quadro 1 - Cronograma de talleres abiertos}

\begin{tabular}{ccccc}
\hline Lunes & Martes & Miercoles & Jueves & Viernes \\
15:00 Origami & $\begin{array}{c}15: 30 \\
\text { Periodismo }\end{array}$ & $\begin{array}{c}15: 00 \\
\text { Plástica }\end{array}$ & $\begin{array}{c}15.15 \text { Trabajo } \\
\text { corporal }\end{array}$ & 15:00 Teatro \\
\hline
\end{tabular}

Fonte/créditos: Produzido pelos autores.

\footnotetext{
${ }^{9}$ También aquí es necesario un reconocimiento a la enorme generosidad de talleristas que concurren al hospital y se brindan en un trabajo a puro gasto, es decir, totalmente sostenido en el deseo.

${ }^{10}$ Podría tomarse como un primer intento de soporte topológico para este espacio particular a la botella de Klein.
} 
En este momento nos encontramos próximos a la inauguración de una Biblioteca. La misma fue pensada en forma conjunta entre el equipo y algunos pacientes interesados en el tema. Fue concebida también como un espacio abierto y resultaron enriquecedores el encuentro y articulación con la escuela domiciliaria. Se pone a disposición de la comunidad no solo el recurso de los libros sino también una pequeña sala de lectura donde proyectamos comenzar en breve con algunas actividades.

\section{FRAGMENTOS ${ }^{11}$}

- Denominamos VOZ a aquello que excede a la estructura.

- Contar con lo que excede: La única posibilidad de la libertad (y la única posibilidad de la posibilidad).

- Lo que excede a la estructura: la Voz. También: el Pueblo. Y también: la Clínica.

- Los nombres de lo que excede. Los nombres de los que exceden.

- Pienso fundamentalmente en pacientes. Esos que exceden a la Institución (a la estructura). Me vienen a la cabeza nombres. Todos conocemos los nombres de algunos que exceden a la estructura. Esos que "no son pacientes para este hospital". Frase que miente dando a entender que habría un lugar, una institución, un dispositivo, adecuados. Sabemos que no lo hay. La Clínica aparece como lo que no encaja, lo que no obedece, no se adecúa, no tiene lugar en la estructura.

- Resiste a los intentos de hacerla entrar en caja. A los forzamientos.

- Resiste, desobediente.

- Pensar el dispositivo que no hay y que además no puede haber.

- La única homogeneidad dada por el exceder. El dispositivo para lo que resiste a entrar. Dispositivo situado por fuera. El no dispositivo. "una manera de situar un punto" y no un método que pudiera ser aplicado. Situar un punto para alguien y dejarlo. Soltar-lo. Liberar-lo.

- lo que refiere al punto y al alguien.

- El trabajo por fuera del dispositivo.

${ }^{11}$ Fragmentos de Conversasiones mantenidas en el curso: "Fundamentos de Hospital de Día. Una política". 2015. 
- Dejar el dispositivo.

- Que esté, pero para dejarlo. Trabajar por fuera, con lo que no entra. Con los que no entran.

- Pensar lo que excede en cada quien, para cada quien.

\section{REFERENCIAS}

DERRIDA, J.; ROUDINESCO, E. Y mañana que... Editorial Fondo de Cultura Economica. Buenos Aires 2009.

HEIDEGGER, M. El ser y el tiempo. Editorial Fondo de cultura Económica. Buenos Aires, 2009.

IUORNO, R. En el Hospital... Editorial Catálogos. Primera Edición. Buenos Aires, 2004.

NANCY, J-L. La participación de las artes. Edición de Cristina Rodríguez Marciel. Valencia: Pre-Textos-Universidad Politécnica de Valencia, 2013. 